\title{
Perfectly Symmetric Two-Dimensional Integration Formulas with Minimal Numbers of Points
}

\author{
By Philip Rabinowitz and Nira Richter
}

\begin{abstract}
Perfectly symmetric integration formula of degrees 9-15 with a minimal number of points are computed for the square, the circle and the entire plane with weight functions $\exp \left(-\left(x^{2}+y^{2}\right)\right)$ and $\exp \left(-\left(x^{2}+y^{2}\right)^{1 / 2}\right)$. These rules were computed by solving a large system of nonlinear algebraic equations having a special structure. In most cases where the minimal formula has a point exterior to the region or where some of the weights are negative, 'good' formulas, which consist only of interior points and have only positive weights, are given which contain more than the minimal number of points.
\end{abstract}

1. Introduction. Integration rules over a square with a minimal number of points are not too important for the computation of particular definite integrals. Since the computation of the integrand on a larger set of points does not make too much difference for a computer, accurate integration rules are readily available. Among these are the product Gauss rules [4], Romberg integration in two dimensions [1], [6], and iterated integration, where in each dimension, an adaptive scheme is used [7], [8]. However, the number of points in an integration rule is very important when solving a two-dimensional integral equation by numerical integration. kind

Consider for example, the linear homogeneous Fredholm equation of the second

$$
f(x, y)=\lambda \int_{-1}^{1} \int_{-1}^{1} K(x, u, y, z) f(u, z, d u a z,
$$

where $K(x, u, y, z)$ is the kernel of the equation, $\lambda$ is an eigenvalue and $f(x, y)$ an eigenfunction to be determined. Replacing the double integral by a numerical integration rule, we get

$$
\bar{f}(x, y)=\bar{\lambda} \sum_{i=1}^{n} w_{i} K\left(x, x_{i}, y, y_{i}\right) \bar{f}\left(x_{i}, y_{i}\right),
$$

where $w_{i}$ are the weights and $\left(x_{i}, y_{i}\right)$ the 'abscissas' of the given $n$-point integration rule. The solution of $(1.2), \bar{f}(x, y)$, is then an approximation to the desired eigenfunction $f(x, y)$ while $\bar{\lambda}$ is an approximation to the corresponding eigenvalue $\lambda$. Substituting in (1.2) the $n$ 'abscissas' of our integration rule, we get

$$
\bar{f}_{j}=\bar{\lambda} \sum_{i=1}^{n} K_{j i} \bar{f}_{i}, \quad j=1, \cdots, n,
$$

where $\bar{f}_{j} \equiv \bar{f}\left(x_{j}, y_{j}\right), K_{i j} \equiv w_{j} K\left(x_{i}, x_{j}, y_{i}, y_{j}\right)$. Thus, our original problem has been transformed into a linear homogeneous system of $n$ algebraic equations in $n$ unknowns. The solution of this approximation to Eq. (1.1) requires the computation

Received January 3, 1969, revised April 21, 1969. 
of the eigenvalues and eigenvectors of the $n$-dimensional matrix $\left(K_{i j}\right)$. The order of the matrix, $n$, plays an important role in determining the difficulty of the computation since computing time varies as $n^{3}$ and memory space varies as $n^{2}$. Hence, small values of $n$ which give the accuracy needed are desirable.

A second application in which the number of points in an integration rule is critical is when a sequence of functions of two variables is to be orthonormalized by the Gram-Schmidt process. Here, each function is represented by a vector of points, taken to be 'abscissas' of a sufficiently accurate integration rule, since the inner product

$$
(f, g)=\int_{-1}^{1} \int_{-1}^{1} f(x, y) g(x, y) d x d y
$$

is approximated by the sum

$$
(f, g)=\sum_{i=1}^{n} w_{i} f\left(x_{i}, y_{i}\right) g\left(x_{i}, y_{i}\right) .
$$

Again, the amount of computation and storage depends on $n$, although in this case, only linearly.

Hammer and Stroud [3] have given perfectly symmetric integration rules over the square with a minimal number of points which are accurate for polynomials up to degree 7. In this work, formulas will be given which are accurate for polynomials up to degree 15. We point out that there may exist integration rules with fewer number of points which are accurate for polynomials of a particular degree. Thus Radon [10] has given a 7-point formula of 5th degree accuracy while the 5th degree formula in [3] requires 9 points. However, formulas which are not perfectly symmetric are much more difficult to compute if they exist at all. Because the region under consideration is perfectly symmetric, we limit ourselves to perfectly symmetric rules.

Since the method of computation does not depend on the region involved, so long as it is perfectly symmetric, we have also computed integration rules for the unit circle and for the entire plane. In the latter case, suitable perfectly symmetric weight functions were used. The discussion will generally refer to the square but can be applied to the other regions with the obvious changes. In fact, the only real change is in the definition of $I[f(x, y)]$.

In addition to minimal formulas, we give 'good' formulas, which consist only of interior points and have positive weights, in most cases where the minimal formula was not 'good.' These formulas contain more than the minimal number of points. In no case did the minimal formulas computed here have a zero weight. Hence, for the cases considered here, there is no 'subminimal' rule with fewer than the expected minimal number of points needed for consistency with the exactness conditions.

2. Perfectly Symmetric Rules. The square $S$ with vertices $( \pm 1, \pm 1)$ is a perfectly symmetric region, in that, for each point $(x, y)$ in $S$, every point of the form $( \pm x, \pm y)$ and $( \pm y, \pm x)$ belongs to $S$. Perfect symmetry of a set implies thus that it is closed with respect to change of sign and/or permutation of coordinates. Since we are interested in integration rules over $S$, we shall choose our integration points as the union of perfectly symmetric sets, where we shall assign the same 
weight to all points of a given set. Every such set is well defined by one of its points which we shall call its generator. Thus, an integration rule will be given by a set of generators and corresponding weights.

The integration rules given in [3] and [11] consist of points whose generators are of the form $(0,0),(a, 0),(b, b)$. By restricting oneself to points of this form, it is only possible to derive rules of degree $\leqq 7$. For rules of degree $\geqq 8$, it is necessary to include points whose generators are of the form $(a, b)$. To get a rule of degree $k$, we have to find weights and points which satisfy the set of equations necessary to insure that the rule will be exact for all polynomials of degree $\leqq k$.

We introduce the following notation:

$$
I[f(x, y)] \equiv \int_{1}^{1} \int_{1}^{1} f(x, y) d x d y .
$$

Generator

$(0,0)$

$\left(u_{i}, 0\right)$

$\left(v_{i}, v_{i}\right)$

$\left(w_{i}, z_{i}\right)$ $i=1, \cdots, K_{1}$

$i=1, \cdots, K_{2}$

$i=1, \cdots, K_{3}$

\section{Weight}

$p$

$a_{i}$

$b_{i}$

$c_{i}$
Number of points in set 1

4

4

8

With this notation, we get the following set of equations for an integration rule of degree $n$ :

$$
\begin{aligned}
& p+4 \sum_{i=1}^{K_{1}} a_{i}+4 \sum_{i=1}^{K_{2}} b_{i}+8 \sum_{i=1}^{K_{3}} c_{i}=I[1] \\
& 2 \sum_{i=1}^{K_{1}} a_{i} u_{i}{ }^{2 k}+4 \sum_{i=1}^{K_{2}} b_{i} v_{i}{ }^{2 k}+4 \sum_{i=1}^{K_{3}} c_{i}\left(w_{i}{ }^{2 k}+z_{i}{ }^{2 k}\right)=I\left(x^{2 k}\right) \\
& k=1, \cdots,[n / 2], \\
& 4 \sum_{i=1}^{v_{2}} b_{i} v_{i}{ }^{2(j+k)}+4 \sum_{i=1}^{K_{3}} c_{i}\left(w_{i}{ }^{2 k} z_{i}{ }^{2 j}+w_{i}{ }^{2 j} z_{i}{ }^{2 k}\right)=I\left(x^{2 j} y^{2 k}\right), \\
& 1 \leqq j \leqq k ; j+k=2, \cdots,[n / 2] .
\end{aligned}
$$

It is clear that an integration rule of degree $2 m$ is identical to that of $2 m+1$, since both yield the same set of equations. The numbers $K_{1}, K_{2}, K_{3}$ are determined by the form of the system, as we shall see. There are two types of equations in the system. Type I consists of those equations which contain all the unknowns or all except $p$, i.e. Eqs. (2.1), (2.2k). Type II consists of those equations in which the unknowns $p, a_{i}, u_{i}\left(i=1, \cdots, K_{1}\right)$ do not appear. These are Eqs. (2.3jk).

By subtracting from each equation of type II, an equation of type I of the same degree, we get a collection of equations in which the unknowns $p, v_{i}, b_{i}(i=1$, $\cdots, K_{2}$ ) do not appear. We call this set type $\mathrm{II}^{\prime}$. The number of equations of type

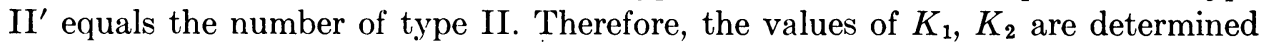
according to the same considerations as regards consistency of the system.

For a rule of degree $n=2 m$, the number of equations of type $\mathrm{I}$ is $l_{1}=m+1$ while that of type II is $l_{2}$ where 


$$
\begin{aligned}
l_{2} & =\left(\frac{m}{2}\right)^{2}, \quad m \text { even }, \\
& =\left[\frac{m}{2}\right] \cdot\left[\frac{m+1}{2}\right]=\frac{(m-1)(m+1)}{4}, \quad m \text { odd } .
\end{aligned}
$$

For $m \geqq 4$, there are at least two equations of type II (II') with the same degree. From $j$ equations of type II ( $\left.\mathrm{II}^{\prime}\right)$ of the same degree, it is possible to get $j-1$ equations in which appear only the variables $c_{i}, w_{i}, z_{i}\left(i=1, \cdots, K_{3}\right)$. These equations belong to type III and number $l_{3}=l_{2}-(m-1)$. Since we have $l_{3}$ equations and $3 K_{3}$ unknowns $c_{i}, w_{i}, z_{i}\left(i=1, \cdots, K_{3}\right)$ the condition $3 K_{3} \geqq l_{3}$ is necessary for the system to be consistent. For $m \geqq 4, l_{3}>0$; hence, for rules of degree $\geqq 8$, $K_{3} \neq 0$ and it is necessary to introduce generators of the type $(a, b)$.

We summarize the classification of equations into types as follows:

1. Type I equations: Contain all the parameters (2.1) or all except $p(2.2 k)$.

2. Type II equations: Do not contain parameters $a_{i}, u_{i}(2.3 j k)$.

3. Type II' equations: Do not contain parameters $b_{i}, v_{i}$.

4. Type III equations: Involve only the parameter $c_{i}, w_{i}, z_{i}$. These are solved first. They are the most troublesome and cause us to restrict ourselves to $m \leqq 7$.

5. Type IV equations: Subset of type II equations with $j=1$. Once $c_{i}, w_{i}$ and $z_{i}$ are computed by solving the set of type III equations, the set of type II equations are not linearly independent. The set of type IV equations is a linearly independent and complete subset.

6. Type $I V^{\prime}$ equations: A similarly defined subset of type $\mathrm{II}^{\prime}$ equations.

7. A 'good' rule: A rule which has positive weights and abscissas lying within the region of integration.

To get rules with a minimal number of points, $K_{3}$ should be the smallest integer which satisfies $3 K_{3} \geqq l_{3}$. This is so since a generator of the form $\left(w_{i}, z_{i}\right)$ contributes 8 points to the integration rule and adds only 3 unknowns, while generators of the form $\left(u_{i}, 0\right),\left(v_{i}, v_{i}\right)$ contribute only 4 points while adding 2 unknowns. Thus $K_{3}$ is uniquely determined. Since the number of equations of type II (II') is $l_{2}, K_{1}$ and $K_{2}$ must satisfy the following conditions:

$$
\begin{aligned}
2 K_{2}+3 K_{3} & \geqq l_{2}, \\
2 K_{1}+3 K_{3} & \geqq l_{2}, \\
K_{0}+2 K_{1}+2 K_{2}+3 K_{3} & =l_{1}+l_{2},
\end{aligned}
$$

where

$$
\begin{aligned}
K_{0} & =1,\left(l_{2}+l_{1}-3 K_{3}\right) \quad \text { odd }, \\
& =0,\left(l_{2}+l_{1}-3 K_{3}\right) \text { even. }
\end{aligned}
$$

The meaning of $K_{0}=1$ is that the point $(0,0)$ which contributes one unknown $p$ is included in the rule.

The following scheme gives an overall picture of the calculation. The details, which differ according as $l_{3}$ is divisible by 3 or not and as $m$ is even or odd, are given in subsequent sections.

1. Consider the system $S_{\text {III }}$ of type III equations.

(a) If $S_{\text {III }}$ is underdefined, assign one or two values $x, y$ as needed. 
(b) Solve $S_{\text {III }}($ Section $5(\mathrm{~b}))$.

2. Consider the system $S_{\text {IV }}\left(S_{\text {IV }}\right)$ of type IV (IV') equations, after substitution of the values obtained in $1(\mathrm{~b})$.

(a) If $S_{\text {IV }}$ is underdefined, assign one value $z$ and proceed to $2(\mathrm{c})$.

(b) If $S_{\mathrm{IV}}$ is overdefined, solve all but one equation and compute the residual $D(x)$ or $D_{1}(x, y)$ - a measure of the margin by which the remaining equation is not satisfied. Proceed to 3.

(c) Solve $S_{\text {IV }}$ (Section 5(a)).

3. Consider the system $S_{\text {I }}$ of type I equations after substitution of the values obtained in 1 (b) and $2(\mathrm{c})$. If there are no assigned values from 1 (a) or 2(a), solve $S_{\text {I }}$ (Section 5(a)) and proceed to 5. Otherwise

(a) Solve all but one equation and compute the residual $D(x), D_{1}(x, y)$, or $D(z)$, according to the assigned values in the previous step. As in 2(b), this residual is a measure of the margin by which the remaining equation is not satisfied.

4. Iterate between 1 (a) or 2 (a) and 3 until the residuals involved vanish.

5. If the solution yields a 'good' rule, terminate the process. Otherwise introduce additional points (Section 6 ) and return to 1.

Sections 3 and 4 indicate the form of $S_{\mathrm{IV}}\left(S_{\mathrm{IV}}\right)$ in the various cases. Section 5 (a) gives an algorithm for solving $S_{\mathrm{IV}}$ and $S_{\mathrm{I}}$ algebraically. Section $5(b)$ gives the form of $S_{\text {III }}$ and the method of solution. Section 6 discusses the addition of points to achieve 'good' rules.

3. Detailed Description of all Calculations. For the solution of the system of equations, it is desirable to distinguish 2 cases:

Case $1 . l_{3}$ is divisible by 3 .

Case $2 . l_{3}$ is not divisible by 3 .

In the present paper, we computed integration formulas for $m=4,5,6,7$. For these values of $m$, Case 1 occurs only for $m=7$ while Case 2 occurs for the other values of $m$.

Solution of the System of Equations for Case $1(m=7)$. Since $\mathrm{K}_{3}=l_{3} / 3$ for Case 1 , the set of equations of type III consists of a system of $l_{3}$ equations in the $l_{3}$ unknowns $c_{i}, w_{i}, z_{i}, i=1, \cdots, K_{3}$. After solving this subsystem, we must solve $m-1$ equations of type II ( $\left.\mathrm{II}^{\prime}\right)$, each one of a different degree.

In this work, we chose as the $m-1$ equations of the set $(2.3 j k)$, those for which $j=1, k=1,2, \cdots,[n / 2]-1$. We call these equations type IV. There is a corresponding set which we call type IV' $^{\prime}$ whose relation to type IV is similar to the relation of type $\mathrm{II}^{\prime}$ to type II. The solution of these $m-1$ equations where the unknowns are $b_{i}, v_{i}, i=1, \cdots, K_{2}\left(a_{i}, u_{i}, i=1, \cdots, K_{1}\right)$ will satisfy all the equations of type II $\left(\mathrm{II}^{\prime}\right)$. Since we must solve $m-1$ equations in $2 K_{2}\left(2 K_{1}\right)$ unknowns, the minimal $K_{2}\left(K_{1}\right)$ possible is $K_{2}=[m / 2]\left(K_{1}=[m / 2]\right)$. For this choice of $K_{2}\left(K_{1}\right), K_{1}\left(K_{2}\right)$ is fully determined and its maximum value is $K_{1}=(m+1) / 2$ $\left(K_{2}=(m+1) / 2\right)$. We thus have the following condition: $[m / 2] \leqq K_{i} \leqq[(m+1) / 2]$, $i=1$, 2. If $m$ is even, $K_{1}=K_{2}=m / 2$ while if $m$ is odd, there are two possibilities:

$$
\begin{array}{ll}
K_{2}=(m+1) / 2, & K_{1}=(m-1) / 2, \\
K_{2}=(m-1) / 2, & K_{1}=(m+1) / 2 .
\end{array}
$$


We must therefore distinguish between the cases $m$ even and $m$ odd.

(a) $m$ odd. The solution can continue in either of two ways, where the alternate way is indicated in parentheses. Choose $K_{2}=(m-1) / 2\left(K_{1}=(m-1) / 2\right)$ and solve the system of $m-1$ equations of type IV (IV') in $m-1$ unknowns, $b_{i}, v_{i}$, $i=1, \cdots, K_{2}\left(a_{i}, u_{i}, i=1, \cdots, K_{1}\right)$. After solving this system, it remains to solve $m+1$ equations of type $\mathrm{I}$ in the $m+1$ unknowns $a_{i}, u_{i}, i=1, \cdots, K_{1}\left(b_{i}, v_{i}, i=\right.$ $\left.1, \cdots, K_{2}\right)$.

(b) $m$ even. (We include this section for the sake of completeness, although this method was never used, since for $m=8$, the attempt to solve the type III equations failed.) The solution is possible in only one way since $K_{1}=K_{2}=m / 2$. The number of equations of type IV is $m-1$ and therefore this is a system in which the number of unknowns is one more than the number of equations. To solve these equations, we employ the following method: To one of the $m$ unknowns from the set $b_{i}, v_{i}$, $i=1, \cdots, K_{2}$ which we shall denote by $x$, we give an arbitrary value. With this value of $x$, we solve the $m-1$ equations for the remaining $m-1$ unknowns. Then $m$ of the $m+1$ equations of type I are solved for the $m$ unknowns $a_{i}, u_{i}, i=1$, $\cdots, K_{1}$. For the remaining equation of type $\mathrm{I}$, the residue $D$ is computed. This residue is a function of $x, D(x)$. We thus have to solve the equation $D(x)=0$ to get a solution to our system. Since the explicit form for $D(x)$ is not known, the equation is solved by bisection, after finding two values $x_{1}, x_{2}$ for which $D\left(x_{1}\right) \cdot D\left(x_{2}\right)<0$.

4. Solution of the System of Equations for Case $2(m=4,5,6)$. For $m=4$, the general scheme described below was not used, since in this case $l_{3}=1$ and hence we could solve directly the set of equations of type II. We thus could solve the system with only one residual to be made zero while the general scheme would have required us to find the zeros of two residuals.

In Case $2, l_{3}$ is not divisible by 3 and therefore $3 K_{3}>l_{3}$. Hence the set of equations of type III cannot be solved directly since the number of unknowns is greater than that of equations. Since $l_{3}=[(m-1) / 2] \cdot[(m-2) / 2]$, we have

$$
\begin{aligned}
3 K_{3}-l_{3} & =1, m \text { odd }, \\
& =2, m \text { even } .
\end{aligned}
$$

The total number of equations of type II $\left(\mathrm{II}^{\prime}\right)$ is $l_{3}+m-1$ while the number of unknowns is $3 K_{3}+2 K_{2}\left(3 K_{3}+2 K_{1}\right)$. Hence the following conditions must hold:

$$
\begin{aligned}
& 3 K_{3}+2 K_{2} \geqq l_{3}+m-1, \\
& 3 K_{3}+2 K_{1} \geqq l_{3}+m-1 .
\end{aligned}
$$

Therefore, the minimal value of $K_{2}\left(K_{1}\right)$ is [ $\left.(m-1) / 2\right]$. For such a choice, $K_{1}\left(K_{2}\right)$ is uniquely determined and takes on its maximum value $[m / 2]$. This follows since the total number of equations in the system is $l_{3}+2 m$ and $K_{1}\left(K_{2}\right)$ satisfies the requirement $K_{0}+3 K_{3}+2 K_{2}+2 K_{1}=l_{3}+2 m\left(K_{0}=1\right.$ for $m$ odd and $K_{0}=0$ for $m$ even). Thus, for $m$ odd, $K_{1}, K_{2}$ are uniquely determined, $K_{1}=K_{2}=$ $(m-1) / 2$, while for $m$ even, there are two choices for $K_{1}, K_{2}$ :

$$
\begin{aligned}
& K_{1}=m / 2, \quad K_{2}=(m-2) / 2, \\
& K_{1}=(m-2) / 2, \quad K_{2}=m / 2 .
\end{aligned}
$$


Hence, we shall again distinguish between the cases $m$ even and $m$ odd.

(a) $m$ odd. The set of equations of type III has $l_{3}$ equations in $l_{3}+1$ unknowns $c_{i}, w_{i}, z_{i}, i=1, \cdots, K_{3}$. We assign to one of the unknowns, $x$, an arbitrary value and then solve the resulting system. We then proceed to solve the set of equations of type IV, which consists of $m-1$ equations in $2 K_{2}$ unknowns, $b_{i}, v_{i}, i=1, \cdots$, $K_{2}$. Since $K_{2}=(m-1) / 2$, this set can be solved. To complete the solution, we must solve the $m+1$ equations of type I where the unknowns are $p, a_{i}, u_{i}, i=1$, $\cdots, K_{1}$. Since $K_{1}=(m-1) / 2$, the number of unknowns, $m$, is less by one than the number of equations and hence we solve only $m$ of the $m+1$ equations. Denoting by $D(x)$, the residual in the remaining equation, we proceed as before to solve $D(x)=0$ by bisection.

(b) $m$ even. This is the most complicated case, and as before, can be treated in two ways. The number of equations of type III is two less than the number of unknowns, and hence we choose two unknowns denoted $x, y$ and assign them arbitrary values. Then we solve the set for the remaining $l_{3}$ unknowns. We next choose $K_{2}=(m-2) / 2\left(K_{1}=(m-2) / 2\right)$ and solve set IV $\left(I^{\prime}\right)$. This set has $m-1$ equations in $m-2$ unknowns $b_{i}, v_{i}, i=1, \cdots, K_{2}\left(a_{i}, u_{i}, i=1, \cdots, K_{1}\right)$. Therefore we can only solve $m-2$ of the equations in this set and for the remaining equation, the equation of $(2.3 \mathrm{kj})$ corresponding to $j=k=1$, (the corresponding equation of type $I V^{\prime}$ ), we compute the residual $D_{1}$. There remains now to solve the $m+1$ equations of type I for the $m$ unknowns $a_{i}, u_{i}, i=1, \cdots, K_{1}\left(b_{i}, v_{i}, i=1\right.$, $\left.\cdots, K_{2}\right)$. Again we solve $m$ of these equations and for the equation not satisfied, Eq. (2.1), we compute the residual $D_{2}$. We thus have $D_{1}=D_{1}(x, y)$ and $D_{2}=$ $D_{2}(x, y)$ and it remains to find a point in the $(x, y)$ plane so that $D_{1}=D_{2}=0$.

This method was used for $m$ odd to compute the integration rule of degree 10 $(m=5)$. For $m=6$, no point $\left(x_{0}, y_{0}\right)$ was found for which $D_{1}\left(x_{0}, y_{0}\right)=D_{2}\left(x_{0}, y_{0}\right)$ $=0$. However, points were found for which $D_{1}(x, y)=0$ and

$$
D_{2}(x, y)=4-4 \sum_{i=1}^{K_{1}} a_{i}-4 \sum_{i=1}^{K_{2}} b_{i}-8 \sum_{i=1}^{K_{3}} c_{i}>0 .
$$

Hence, by the addition of the generator $(0,0)$ which contributes only one point to the integration rule, we could satisfy $(2.1)$ by setting $p=D_{2}(x, y)$. This yielded an integration rule of degree 12 .

5. The Algorithms for the Solution of the Subsystems. (a) In the solution of equations of types IV and I, we have to solve systems of the form

$$
\sum_{i=1}^{n} p_{i} x_{i}{ }^{l+k}=T_{k}, \quad k=0,1, \cdots, 2 n-1
$$

and

$$
p_{0} A^{l+k}+\sum_{i=1}^{n} p_{i} x_{i}{ }^{l+k}=T_{k}, \quad k=0,1, \cdots, 2 n,
$$

where the unknowns are the $p_{i}$ and $x_{i}$ while the $T_{k}$ and $A$ are given. (System (5.2) arises when an arbitrary value is given to one of the unknowns $u_{i}$ or $v_{i}$.) To solve (5.1), we proceed as follows: (cf. Davis and Rabinowitz [2, p. 42]). In step 1, we 
multiply each equation by $x_{1}$ and subtract it from the succeeding one. This yields the following system of $2 n-1$ equations in $2 n-1$ unknowns:

$$
\sum_{i=2}^{n} p_{i} x_{i}^{l+k-1}\left(x_{i}-x_{1}\right)=T_{k}-x_{1} T_{k-1}, \quad k=1, \cdots, 2 n-1 .
$$

At step $j$, we multiply each of the $2 n-j+1$ equations by $x_{j}$ and subtract it from the succeeding equation to get the following system of $2 n-j$ equations in $2 n-j$ unknowns:

$$
\begin{aligned}
& \sum_{i=j+1}^{n} p_{i} x_{i}{ }^{l+k-j}\left(x_{i}-x_{1}\right)\left(x_{i}-x_{2}\right) \cdots\left(x_{i}-x_{j}\right) \\
& =T_{k}-T_{k-1} \sum_{i=1}^{j} x_{i}+T_{k-2} \sum_{i=1: p<i}^{j} x_{i} x_{p}-\cdots+(-1)^{j} T_{k-j}\left(x_{1} x_{2} \cdots x_{j}\right), \\
& \quad k=j, \cdots, 2 n-1 .
\end{aligned}
$$

After $n$ steps, we are left with the following system of $n$ equations in the $n$ unknowns $x_{1}, \cdots, x_{n}$ :

$$
0=T_{k}+\sum_{i=1}^{n}(-1)^{i} T_{k-i} Q_{i}, \quad k=n, \cdots, 2 n-1,
$$

where $Q_{i}$ is the symmetric polynomial of degree $i$ in the $n$ variables $x_{1}, \cdots, x_{n}$.

$$
\begin{aligned}
& Q_{1}=x_{1}+x_{2}+\cdots+x_{n} \\
& Q_{2}=\sum_{i=1: j<i}^{n} x_{i} x_{j} \\
& \cdot \\
& \cdot \\
& Q_{n}=x_{1} x_{2} \cdots x_{n} .
\end{aligned}
$$

The system (5.3) is a linear system of $n$ equations in the $n$ unknowns $Q_{1}, \cdots, Q_{n}$ which can be solved by Gaussian elimination. Having computed the $Q_{i}$, we now find the roots of the polynomial

$$
P_{n}(x)=x^{n}+\sum_{i=1}^{n}(-1)^{i} Q_{i} x^{n-i} .
$$

This yields the desired roots of $(5.1), x_{1}, \cdots, x_{n}$.

To find values for $p_{1}, \cdots, p_{n}$, we can substitute the values of $x_{1}, \cdots, x_{n}$ into (5.1) and solve a set of linear equations by the usual methods. However, it is possible to benefit from the special form of the system to compute the $p_{i}$ directly. At step $n-1$ in the reduction of system (5.1), we have equations of the form

$$
p_{n} x_{n}{ }^{l}\left(x_{n}-x_{1}\right)\left(x_{n}-x_{2}\right) \cdots\left(x_{n}-x_{n-1}\right)=T_{n-1}-\sum_{i=1}^{n-1}(-1)^{i} Q_{i}{ }^{(n)} T_{n-1-i}
$$

where $Q_{i}{ }^{(n)}$ is the symmetric polynomial of degree $i$ in the $n-1$ variables $x_{1} \cdots x_{n-1}$. From this equation, one can compute the value of $p_{n}$, given the values of $x_{1}, \cdots, x_{n}$. Since each pair $p_{i}, x_{i}$ has the same role in (5.1), we can compute all the $p_{j}$ by interchanging subscripts as follows: 


$$
p_{j}=\frac{T_{n-1}-\sum_{i=1}^{n-1}(-1)^{i} T_{n-i-i} Q_{i}{ }^{(j)}}{\prod_{i=1 ; i \neq j}^{n}\left(x_{j}-x_{i}\right)}
$$

where $Q_{i}{ }^{(j)}$ is the symmetric polynomial of degree $i$ in the $n-1$ variables $x_{1}$, $x_{2}, \cdots, x_{j-1}, x_{j+1}, \cdots, x_{n}$.

By multiplying each equation of system (5.2) by $A$ and subtracting it from the succeeding equation, we can bring (5.2) to the form of (5.1). We then have a system of $2 n$ equations in $2 n$ unknowns $x_{1}, \cdots, x_{n}, \bar{p}_{1}, \cdots, \bar{p}_{n}$ where $\bar{p}_{i}=p_{i}\left(x_{i}-A\right)$.

(b) The first stage in the solution of the original system of equations involves the solution of a set of type III. For $m=4,5,6,7$ the number of such equations to be considered is only $1,2,4,6$ respectively. This set results from the elimination of one equation of each degree from the set of type II. This elimination can be done in various ways. In our method, the following equations resulted:

$$
\begin{aligned}
& \sum_{i=1}^{K_{3}} c_{i}\left(w_{i}{ }^{2}-z_{i}{ }^{2}\right)^{2}\left(w_{i}{ }^{2} z_{i}{ }^{2}\right)^{j}\left(w_{i}{ }^{2}+z_{i}{ }^{2}\right)^{k}=a_{j k}, \\
& j=1, \cdots,[(m-2) / 2], \quad k=0,1, \cdots, m-2 j-2,
\end{aligned}
$$

where

$$
a_{j k}=\frac{1}{8} I\left[\left(x^{2}-y^{2}\right)^{2}\left(x^{2} y^{2}\right)^{j}\left(x^{2}+y^{2}\right)^{k}\right] .
$$

$a_{j k}$ can be readily expressed in terms of the known values of $I\left[x^{2 s} y^{2 t}\right]$.

By setting $x_{i}=w_{i}{ }^{2}+z_{i}{ }^{2}, p_{i}=c_{i}\left(w_{i}{ }^{2}-z_{i}{ }^{2}\right) w_{i}{ }^{2} z_{i}{ }^{2}$, the $m-3$ equations of (5.4) for $j=1$ take the form of (5.1) and can be treated as above. Knowing these values of $x_{i}, p_{i}$, the $m-5$ equations of (5.4) for $j=2$ are linear in the unknowns $\left(w_{i}{ }^{2} z_{i}{ }^{2}\right)$ and readily solvable. For $m=6,7$, equations of type III exist for $j=1,2$ only so that we can get values of $w_{i}{ }^{2} z_{i}{ }^{2}$ and $w_{i}{ }^{2}+z_{i}{ }^{2}$. By solving a quadratic equation, we find $w_{i}, z_{i}$ and then from $p_{i}$, we find $c_{i}$. For $m=5$, equations of type III exist only for $j=1$. There are two equations and three unknowns. Hence as mentioned in Section 4(a), we take an arbitrary value for $z_{1}$ and solve for the other two unknowns. For $m \geqq 8$, system III could not be solved by the above method nor was any other method found.

6. Additional Points. Since we were interested in 'good' integration rules with positive weights and with points within the region of integration, we added points to the integration rule in those cases where either a real solution did not exist or where the minimal rule had external points or negative weights. This yielded 'good' rules although with more than the minimal number of points. The addition of generators was done in such a manner as to minimize the total number of points in the rule as follows:

(a) To rules which did not contain the generator $(0,0)$ it was added, thus adding only one point while contributing one free parameter. In rules which did contain $(0,0)$ it was replaced by a generator of type $(v, 0)$ or $(v, v)$. This added three points while also contributing one free parameter.

(b) If step (a) was not successful, the following device was attempted for rules without $(0,0)$. Two generators of type $(u, 0)$ or $(v, v)$ were replaced by the generators $(0,0)$ and $(w, z)$. This increased the number of points by one, while remaining with 
a system of $n$ equations in $n$ unknowns. For rules with the point $(0,0)$, it and a generator of type $(u, 0)$ or $(v, v)$ were replaced by a generator of type $(w, z)$. This added three points while again remaining with a system of $n$ equations in $n$ unknowns.

(c) If the above actions were unsuccessful, then the minimum number of additional points was 4 . This occurred either by the addition of a generator contributing 4 points to the integration rule and 2 free parameters or by the exchange of a generator contributing 4 points by one contributing 8 , yielding one free parameter. One could, of course, continue to add points in case of failure, at each stage adding a minimal number of points. However, there is a limit to this process insofar as it concerns the efficiency of the resulting integration rules. Since the product of two $m$-point Gauss integration rules requires $m^{2}$ points and is accurate for all polynomials up to degree $2 m-1$, only rules of degree $2 m-1$ requiring less than $m^{2}$ points are of interest.

7. Summary of Integration Rules Computed. (a) For the square, the following rules were computed and are listed in Table 1 . For degree 9, a 'good' rule with the minimal number of points was found. This rule uses 20 points while the product Gauss rule of degree 9 requires 25 points. For degree 11, the minimal integration rule of 25 points had one generator exterior to the square. Upon the addition of 3 more points, a 'good' rule was found with 28 points while the corresponding Gauss rule has 36 points. For degree 13, no rule containing the minimal number of points was found. However, upon the addition of the origin, a 'good' rule with 37 points was computed. The corresponding Gauss rule consists of 49 points. Finally for degree 15 , the minimal rule of 44 points had one exterior generator. A 'good' rule with 48 points was found as against 64 for the corresponding Gauss rule.

(b) For the unit circle, the following rules were computed and are listed in Table 2. For degree 9 , the minimal rule of 20 points had an exterior generator. A 'good' rule with 21 points was found. For degree 11, two 'good' formulas with 28 points, 3 more than the minimum, are given. For degree 13, the 'good' formula has 37 points as in the case of the square while for degree 15, the 'good' formula has the minimal number of points, 44 . Some of these formulas, namely, the degree 9 formula with 21 points, a degree 11 formula, and the degree 15 formula are given in Krylov and Sul'gina [5]. They were computed in a different fashion and partial details are given by Mysovskih [9]. A different degree 9 formula with 21 points is given in [3].

(c) For integrals over the entire plane, two sets of rules were computed corresponding to the weight functions

$$
\exp \left(-\left(x^{2}+y^{2}\right)\right) \text { and } \exp \left(-\left(x^{2}+y^{2}\right)^{1 / 2}\right)
$$

respectively. For these weight functions, rules up to degree 7 are given by Stroud and Secrest [11]. For both weight functions, rules were computed for degrees 9 to 15 and are listed in Tables 3 and 4. For degrees 9 and 15, the number of points required for 'good' rules was minimal. For degree 11, two 'good' rules are given in each case, requiring 28 points. For degree 13, rules using 37 points are given but in both cases one weight is negative. Since degree 13 is the most difficult case to compute, we did not pursue the matter further. 
TABLE 1

Formulas for the Square

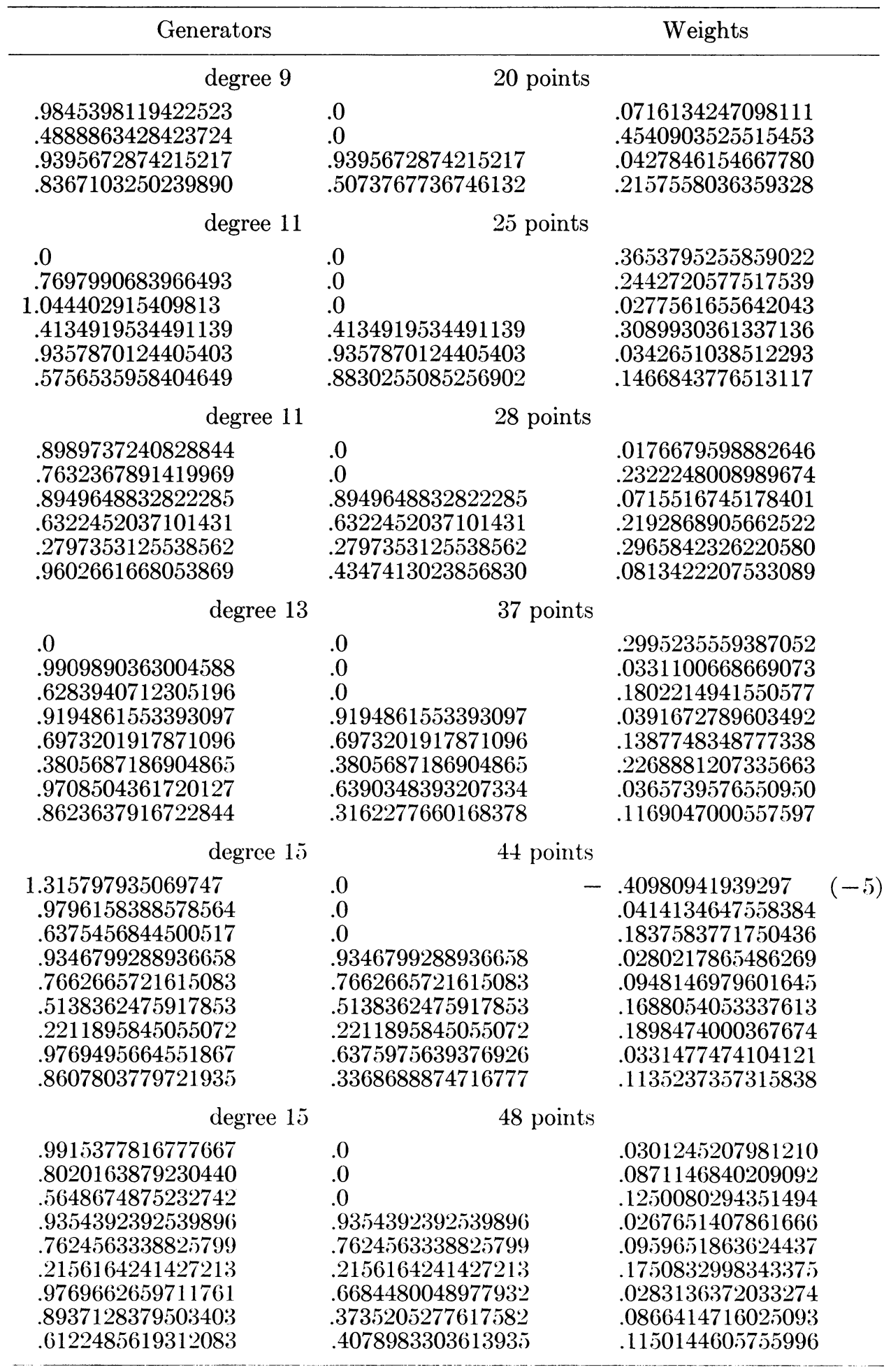


TABLE 2

Formulas for the Circle

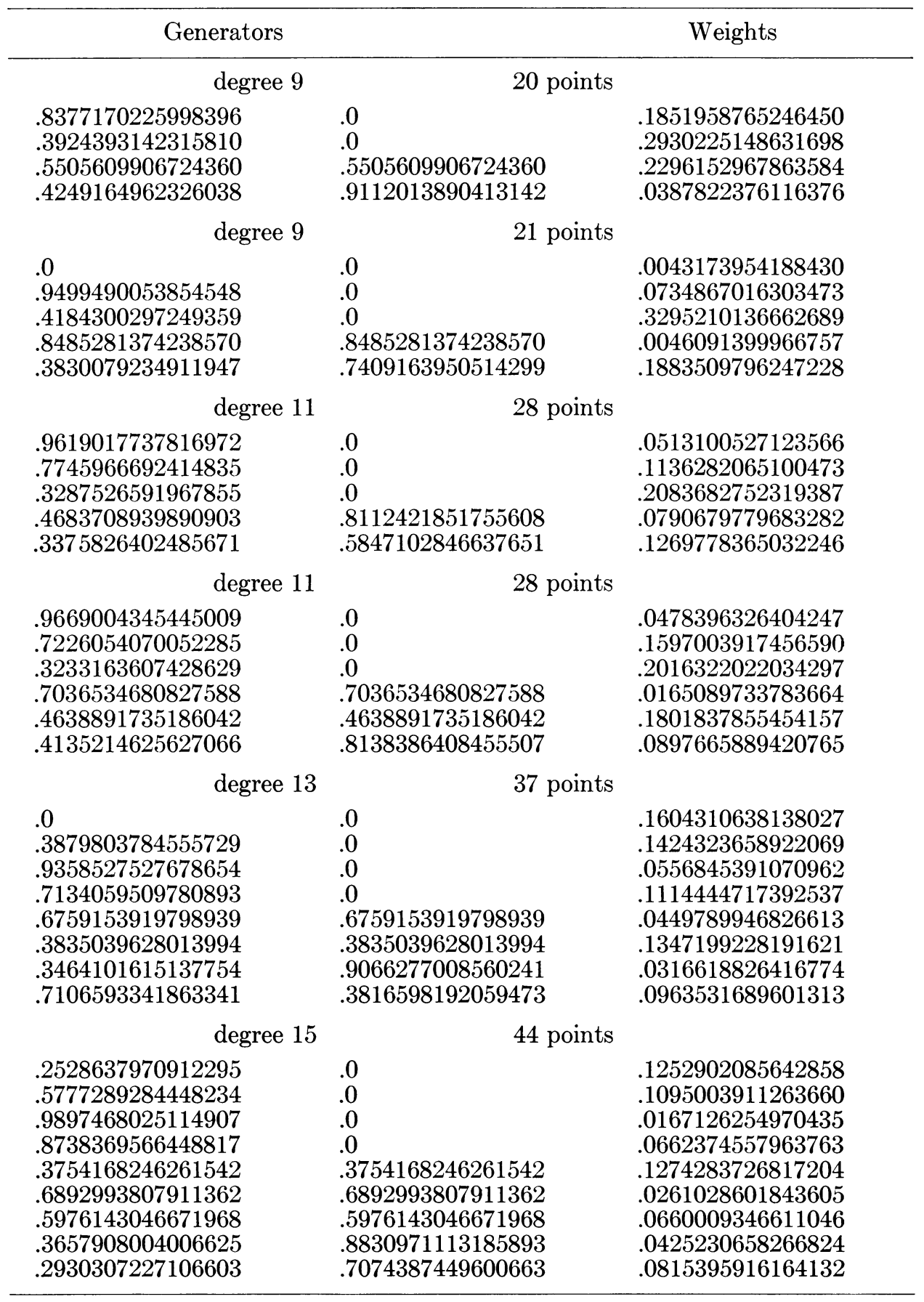


TABLE 3

Formulas for the Plane with Weight Function: $\exp \left[-\left(x^{2}+y^{2}\right)\right]$

\begin{tabular}{|c|c|c|}
\hline Generators & & Weights \\
\hline \multicolumn{2}{|l|}{ degree 9} & \\
\hline $\begin{array}{l}1.538189001320852 \\
1.224744871391589 \\
0.4817165220011443 \\
2.607349811958554\end{array}$ & $\begin{array}{l}0.0 \\
1.224744871391589 \\
0.4817165220011443 \\
0.9663217712794149\end{array}$ & $\begin{array}{l}.1237222328857347 \\
.6544984694978697(-1) \\
.5935280476180875 \\
.1349017971918148(-2)\end{array}$ \\
\hline \multicolumn{2}{|l|}{ degree 11} & \\
\hline $\begin{array}{l}2.757816396257008 \\
1.732050807568877 \\
0.6280515301597559 \\
1.224744871391589 \\
0.7071067811865475\end{array}$ & $\begin{array}{l}0.0 \\
0.0 \\
0.0 \\
2.121320343559643 \\
1.224744871391589\end{array}$ & $\begin{array}{l}.8176645817675417(-3) \\
.4363323129985824(-1) \\
.5373255214498174 \\
.3636102608321520(-2) \\
.9817477042468103(-1)\end{array}$ \\
\hline \multicolumn{2}{|r|}{28 points } & \\
\hline $\begin{array}{l}2.907364117106118 \\
1.528230917660483 \\
0.6178819071436261 \\
1.904162039910276 \\
0.9724173472297303 \\
2.061552812808830\end{array}$ & $\begin{array}{l}0.0 \\
0.0 \\
0.0 \\
1.904162039910276 \\
0.9724173472297303 \\
0.8660254037844387\end{array}$ & $\begin{array}{l}.4106569066965604(-3) \\
.9065690889492120(-1) \\
.5266955729327722 \\
.9681125175723808(-3) \\
.1515812331366514 \\
.7542839504417270(-2)\end{array}$ \\
\hline \multicolumn{2}{|l|}{ degree 13} & \\
\hline $\begin{array}{l}0.0 \\
2.403151765001966 \\
1.298479973315986 \\
1.912428205769905 \\
0.9478854439698223 \\
0.3188824732576547 \\
3.325657829663178 \\
1.882228401823884\end{array}$ & $\begin{array}{l}0.0 \\
0.0 \\
0.0 \\
1.912428205769905 \\
0.9478854439698223 \\
0.3188824732576547 \\
1.145527285699371 \\
0.8826073082889659\end{array}$ & $\begin{array}{l}.7482913219380363 \\
.3521509661098668(-2) \\
.1650055872539264 \\
.8537825937946404(-3) \\
.1326938806789336 \\
.6447719928481539 \\
.1799266413507747(-4) \\
.1279412775888998(-1)\end{array}$ \\
\hline \multicolumn{2}{|c|}{ degree 15} & \\
\hline $\begin{array}{l}3.538388728121807 \\
2.359676416877929 \\
1.312801844620926 \\
0.5389559482114205 \\
2.300279949805658 \\
1.581138830084189 \\
0.8418504335819279 \\
2.685533581755341 \\
1.740847514397403\end{array}$ & $\begin{array}{l}0.0 \\
0.0 \\
0.0 \\
0.0 \\
2.300279949805658 \\
1.581138830084189 \\
0.8418504335819279 \\
1.112384431771456 \\
0.7210826504868960\end{array}$ & $\begin{array}{l}.8006483569659628(-5) \\
.3604577420838264(-2) \\
.1187609330759137 \\
.4372488543791402 \\
.3671735075832989(-4) \\
.5654866776461627(-2) \\
.1777774268424240 \\
.2735449647853290(-3) \\
.2087984556938594(-1)\end{array}$ \\
\hline
\end{tabular}

8. Examples. We tested the various rules for a square on four functions and compared the errors with those obtained by using Gaussian product rules. The results are listed in Table 5 where we have used the notation $M_{p}$ to denote a minimal rule with $p$ points and $G_{n}{ }^{2}$, the product of 2 Gauss $n$-point rules. These examples seem to indicate that not only is a product Gauss rule of given degree superior to other formulas of that degree but also that in many cases, a Gauss rule of lower 
degree is equal if not superior to a minimal rule of higher degree. This is due to the fact that a product Gauss rule of degree $n$ integrates exactly all monomials $x^{i} y^{j}$ for which $i \leqq n, j \leqq n$ while the two-dimensional rules of degree $n$ integrate exactly all monomials $x^{i} y^{j}$ for which $i+j \leqq n$. Because of this, it turns out that the number of points in rules of comparable accuracy is about the same.

TABLE 4

Formulas for the Plane with Weight Function: $\exp \left[-\left(x^{2}+y^{2}\right)^{\frac{1}{2}}\right]$

\begin{tabular}{|c|c|c|}
\hline \multicolumn{2}{|c|}{ Generators } & Weights \\
\hline \multicolumn{2}{|c|}{ degree 9} & 20 points \\
\hline 6.822859174233539 & 0.0 & $.3380228176732269(-1)$ \\
\hline 1.901350903458987 & 0.0 & $.1467201651910359(+1)$ \\
\hline 4.260195453867070 & 4.260195453867070 & $.6973178170307865(-1)$ \\
\hline 6.693991707281686 & 14.77112509749386 & $.3030570706813315(-4)$ \\
\hline \multicolumn{2}{|c|}{ degree 11} & 28 points \\
\hline 12.74800100302598 & 0.0 & $.1528937836199174(-3)$ \\
\hline 6.548756194884845 & 0.0 & $.2460475747386993(-1)$ \\
\hline 1.760536818970077 & 0.0 & $.1409433533958677(+1)$ \\
\hline 10.05412033203744 & 5.804749080166705 & $.4416296048062511(-3)$ \\
\hline 4.616780734333329 & 2.665499599756826 & $.6786094118455858(-1)$ \\
\hline \multicolumn{2}{|c|}{ degree 11} & \\
\hline 13.23694157142503 & 0.0 & $.1020154285801705(-3)$ \\
\hline 5.858647139727296 & 0.0 & $.5959360016181913(-1)$ \\
\hline 1.719290407899388 & 0.0 & $.1389898268451152(+1)$ \\
\hline 12.76644300362842 & 12.76644300362842 & $.1691597241187992(-5)$ \\
\hline 3.556098987915152 & 3.556098987915152 & .1189929098056537 \\
\hline 9.300537618869137 & 4.847679857416328 & $.1103920675225255(-2)$ \\
\hline \multicolumn{2}{|c|}{ degree 13} & \\
\hline 0.0 & 0.0 & $.3497776022412480(+1)$ \\
\hline 19.67638186041246 & 0.0 & $.4425802565915590(-6)$ \\
\hline 8.770037945037203 & 0.0 & $.4553409712395994(-2)$ \\
\hline 10.20568519238436 & 10.20568519238436 & $.2775303265875652(-4)$ \\
\hline 3.591105603680783 & 3.591105603680783 & $.3312777924884182(+1)$ \\
\hline 3.242171893025389 & 3.242171893025389 & $.1010440929995067(+1)$ \\
\hline 11.94169301540818 & 4.911904665577694 & $.1127213703086534(-3)$ \\
\hline 3.287383483530638 & 3.162277660168379 & $.4921143017387419(+2)$ \\
\hline \multicolumn{2}{|c|}{ degree 15} & \\
\hline 19.97643084360520 & 0.0 & $.1783029629694328(-6)$ \\
\hline 11.52881449694446 & 0.0 & $.3075756711058412(-3)$ \\
\hline う.150382368000088 & 0.0 & $.8468502916013910(-1)$ \\
\hline 1.610748055769942 & 0.0 & $.1334535254221420(+1)$ \\
\hline 12.91466976228591 & 12.91466976228591 & $.7736736266035205(-6)$ \\
\hline 7.598036758945039 & 7.598036758945039 & $.5762989342268486(-3)$ \\
\hline 3.275323454134366 & 3.275323454134366 & .1439495304734647 \\
\hline 14.96412806506222 & 6.198344793636629 & $.5384883122895214(-5)$ \\
\hline 8.095727497543633 & 3.353360126759371 & $.3365458295852239(-2)$ \\
\hline
\end{tabular}


In addition, all the rules were tested to see to what accuracy the polynomials, for which they were supposed to be exact, were integrated. Using the listed weights and abscissas and floating point arithmetic correct to over 18 decimal figures, we found the largest relative error to be less than $10^{-12}$.

\section{TABLE 5.}

Errors in Integration of Various Functions by Minimal Rules with $p$ Points, $M_{p}$, and by Products of $n$-Point Gauss Rules, $G_{n}{ }^{2}$.

\begin{tabular}{ccccc}
\hline Rule $\backslash$ Function & $e^{x y}$ & $(1-x y)^{-1}$ & $\sin \left(\frac{1}{2} \pi(x+2 y)\right)$ & $\left((x+1)^{2}+(y+2)^{3}\right)^{-1}$ \\
\hline$M_{20}$ & $2.3(-8)$ & $1.1(-2)$ & $2.6(-7)$ & $1.1(-9)$ \\
$G_{5}{ }^{2}$ & $1.1(-13)$ & $2.6(-2)$ & $2.2(-8)$ & $6.8(-11)$ \\
$M_{25}$ & $1.9(-10)$ & $1.0(-2)$ & $1.3(-9)$ & $2.3(-12)$ \\
$M_{28}$ & $3.0(-10)$ & $2.9(-2)$ & $2.7(-9)$ & $6.3(-12)$ \\
$G_{6}{ }^{2}$ & $<10^{-15}$ & $1.9(-2)$ & $1.0(-10)$ & $1.1(-13)$ \\
$M_{37}$ & $1.4(-12)$ & $2.3(-2)$ & $1.8(-11)$ & $1.9(-13)$ \\
$G_{7}{ }^{2}$ & $<10^{-15}$ & $1.3(-2)$ & $3.6(-13)$ & $1.0(-14)$ \\
$M_{44}$ & $3.8(-14)$ & $1.8(-2)$ & $1.1(-13)$ & $4.5(-14)$ \\
$M_{48}$ & $1.8(-14)$ & $1.8(-2)$ & $9.4(-14)$ & $4.9(-14)$ \\
$G_{8}{ }^{2}$ & $<10^{-15}$ & $1.1(-2)$ & $8.0(-16)$ & $8.0(-17)$ \\
\hline
\end{tabular}

Acknowledgement. The authors wish to thank the referee for his many valuable suggestions which have contributed so much to the readibility of this paper.

Department of Applied Mathematics

The Weizmann Institute of Science

Rehovot, Israel

1. E. B. Anders, "An extension of Romberg integration procedures to $N$-variables," $J$. Assoc. Comput. Mach., v. 13, 1966, pp. 505-510. MR 34 \#3783.

2. P. J. Davis \& P. Rabinowitz, Numerical Integration, Blaisdell, Waltham, Mass., 1967. MR 35 \#2482.

3. P. C. Hammer \& A. H. Stroud, "Numerical evaluation of multiple integrals. II," $M T A C$, v. 12,1958 , pp. $272-280$. MR $21 \# 970$.

4. P. C. Hamm Er \& A. W. Wymore, "Numerical evaluation of multiple integrals. I," $M T A C$, v. 11,1957 , pp. 59-67. MR 19,323.

5. V. I. KRYLOV \& L. T. SUL'GINA, Handbook of Numerical Integration, "Nauka," Moscow, 1966. (Russian) MR 35 \#3879.

6. P.-J. LAuRent, "Formules de quadrature approchée sur domaines rectangulaires convergentes pour toute fonction intégrable Riemann," C. R. Acad. Sci. Paris, v. 258, 1964, pp. 798801. MR $28 \# 3540$.

7. W. M. McKeеman, "Algorithm 146, multiple integration," Comm. ACM, v. 5, 1962, pp. 604-605.

8. W. M. McKeeman, "Algorithm 198, adaptive integration and multiple integration," Comm. $A C M$, v. 6. 1963 , pp. 443-444.

9. I. P. Mysovskin, "On the construction of cubature formulae for the simplest regions," Z. Vyčisl. Mat. i Mat. Fiz., v. 4, 1964, pp. 3-14. (Russian) MR 28 \#3547.

10. J. Radon, "Zur mechanischen Kubatur," Monatsh. Math., v. 52, 1948, pp. 286-300. MR 11,405

11. A. H. Stroud \& I). Secrest, "Approximate integration formulas for certain spherically symmetric regions," Math. Comp., v. 17, 1963, pp. 105-135. MR 28 \#4677. 